\title{
Wireless Mobile Evolution to 4G Network
}

\author{
Mohammed Jaloun, Zouhair Guennoun \\ ${ }^{1}$ Telecoms Engineer - Motorola, Morocco \\ ${ }^{2}$ Laboratoire d'Electronique et de Communications - Rabat, Morocco \\ E-mail:jaloun@googlemail.com,zouhair@emi.ac.ma
}

Received January 30, 2010; revised February 22, 2010; accepted February 24, 2010

\begin{abstract}
In this paper, we give an overview of the evolution of wireless mobiles starting from the first generation which is the analogue system started in 1980's, and passing through the $2 \mathrm{G}$ technologies which are all digital networks and GSM is the most popular network. $2.5 \mathrm{G}$ networks will introduce the packet notion on the existing $2 \mathrm{G}$ networks and $3 \mathrm{G}$ will bring the quality of service as new perspective for the $3 \mathrm{G}$ partnership projet $3 \mathrm{GPP}$ and 3GPP2. However, high demand on data speed has pushed the operators to looking for $3.5 \mathrm{G}$ system. 3.99G networks are defined as long-term evolution for the existing $3 \mathrm{G}$ network and it will be based on OFDM and MIMO technologies. 4G networks was not yet defined, but requirement is to support heterogeneous networks at $100 \mathrm{Mbps}$ mobility data speed.
\end{abstract}

Keywords: 4G, OFDM, MIMO

\section{Introduction}

Mobile networks have evolved through more than three generations, starting with the analogue or first-generation (1G) networks deployed in the early 1980s, and moving on to the digital second-generation $(2 \mathrm{G})$ networks deployed in the early 1990s. Operators started to deploy 3 G networks in 2001-03, and 3.5G networks from around 2005. Networks still in the design phase include $3.9 \mathrm{G}$ and $4 \mathrm{G}$ systems, which are expected to be deployed in the 2008-10 and 2010-20 timeframes, respectively.
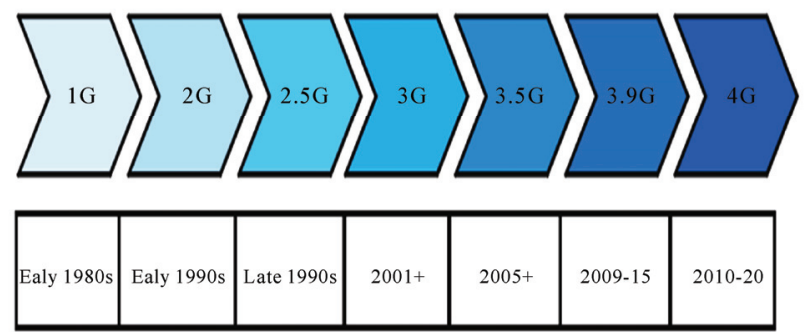

The general principle behind this grouping is that mobile technologies are in the same generation if they have similar network characteristics and deployment timelines.

The International Telecommunications Union (ITU), for example, uses a different approach when defining $3 \mathrm{G}$, it groups technologies based on theoretical maximum connection speeds.
The Table 1 bellows classify networks by generation based on the speed offered:

Notes: WiMAX is classified separately as it has a distinct evolution, but its current capabilities are similar to $3.5 \mathrm{G}$ and its core technologies will be used in $3.9 \mathrm{G}$ systems.

\section{1G Networks}

The first cellular mobile phone systems were introduced in the early 1980s based on analogue standards. Analogue systems are in the process of being phased out, with 1.9 million subscribers worldwide at the end of 2006, down from 4.7 million at end-2005, as they are being superseded by digital networks.

The main analogue systems are:

1) Nordic Mobile Telephone (NMT) was the first operational analogue system in use and was originally introduced into the Nordic countries of Denmark, Finland, Norway and Sweden. NMT runs at $450 \mathrm{MHZ}$ and $900 \mathrm{MHz}$, some NMT 450 networks are still in operation, mainly in Sweden, Russia and Eastern European countries, but most NMT 900 networks have now been closed.

2) Advanced Mobile Phone System (AMPS) has been in operation in North and Latin America since the 1980s. AMPS operates in the $800 \mathrm{MHz}$ frequency band and uses frequency division multiple access (FDMA) technology, which allocates individual frequencies to 
each call, in contrast to other access technologies, which use different methods of dividing up the available spectrum.

3) Total Access Communications System (TACS) is derived from AMPS and was introduced as the analogue standard for the UK operating at $900 \mathrm{MHz}$. Among others, it was also used in China and Japan, which introduced a Japanese Total Access Communications (JTAC) system operating between $800 \mathrm{MHz}$ and $900 \mathrm{MHz}$ ranges.

4) Cellular Digital Packet Data (CDPD) was specified by a consortium of American cellular operators in 1993 and is based on IP overlaying an AMPS network. CDPD makes use of excess capacity on the AMPS network to provide packetised connections up to $19.2 \mathrm{kbps}$, although inherent data overheads reduce this to a practical operating data rate of around $10 \mathrm{kbps}$.

\section{2G Networks}

The majority of mobile phone systems today are based on a number of digital technology networks, and their variants, as defined in Table 2 namely:

1) Global System for Mobile Communication (GSM)

2) Code Division Multiple Access (CDMA)

3) Time Division Multiple Access (TDMA)

4) Integrated Digital Enhanced Network (iDEN)

5) Personal Digital Cellular (PDC)
6) Personal Handyphone System (PHS).

The most important difference between $2 \mathrm{G}$ networks is the spectrum efficiency and the number of channels used as show in Figure 1.

For iden, TDMA and GSM the channel bandwidth is small, it is from $25 \mathrm{KHz}$ for iDEN and TETRA to 1.25 $\mathrm{MHz}$ for CDMA.

Frequency reuse is technically used for TDMA networks, contrarily to CDMA which use the entire spectrum and minimize the interference which allow 1 frequency reuse.

IDen use a small spectrum, because it utilizes a high modulation QAM 16 and QAM 64 and QPSK.

It is easier to understand $2 \mathrm{G}$ Digital networks if it we compared the different multiple access technologies.

Multiple Access Comparison - FDMA

FDMA is used for analog cellular, in this case each user is assigned a discreet slice of spectrum and each user uses 100\% of the channel as show in Figure 2.

\section{Multiple Access Comparison - TDMA}

In Figure 3, TDMA is used for both GSM and IS-54, Key point to note in case of TDMA is that the users are still assigned a discreet slice of RF spectrum, but in this case each RF carrier is further sub-divided into number of time slots, and multiple users sharing the same RF carrier. In case of GSM, 8 users simultaneously transmitting and receiving information on a single carrier, which is 200 $\mathrm{KHz}$ wide.

Table 1. Network generation.

\begin{tabular}{|c|c|c|}
\hline Generation & Data speed* & Networks \\
\hline $1 \mathrm{G}$ & $5-9 \mathrm{kbps}$ & AMPS, NMT, TACS \\
\hline $2 \mathrm{G}$ & 9.6-30 kbps & GSM, CDMA, TDMA, iDEN, PDC, PHS \\
\hline $2.5 \mathrm{G}^{+}$ & $20-130 \mathrm{kbps}$ & GPRS, HSCSD, EDGE**, CDMA2000 $1 \times$ RTT \\
\hline $3 \mathrm{G}$ & $300-600 \mathrm{kbps}$ & WCDMA, CDMA2000 $1 \times$ EV-DO \\
\hline $3.5 \mathrm{G}$ & 3.1-73.5 $\mathrm{Mbps}^{\wedge}$ & $\begin{array}{l}\text { HSDPA, HSUPA, UMTS TDD(TD-CDMA), TD-SCDMA, EV-DO Revision A, } \\
\text { EV-DO Revision B }\end{array}$ \\
\hline $3.9 \mathrm{G}$ & $100-200 \mathrm{Mbps}^{\wedge}$ & 3 GPP LTE, 3 GPP2 UMB \\
\hline $4 \mathrm{G}$ & $100-1000 \mathrm{Mbps}^{\wedge}$ & $\begin{array}{l}\text { Networks undefined but goals include } 100 \mathrm{Mbps} \text { with full mobility and } 1 \mathrm{Gpbs} \text { for } \\
\text { fixed or nomadic access }\end{array}$ \\
\hline
\end{tabular}

Table 2. 2G system.

\begin{tabular}{|c|c|c|c|c|c|c|}
\hline Generation & Network & Launch & Frequencies(MHZ) & Peak data rates & $\begin{array}{c}\text { Average data } \\
\text { Main }\end{array}$ & Main Service \\
\hline \multirow[t]{6}{*}{$2 \mathrm{G}$} & GSM & 1991 & $\begin{array}{c}4001450 ; 800 ; 900 \\
1800 ; 1900\end{array}$ & 9.6-14.4 Kbps & $9.6 \mathrm{Kbps}$ & Voice + data \\
\hline & TDMA & 1993 & $800 ; 1900$ & $19.2 \mathrm{Kbps}$ & 9.6-14.4 Kbps & Voice + data \\
\hline & PDC/PDC-P & 1993 & $800 ; 1500$ & 9.6-28.8 Kbps & $5.6-9.6 \mathrm{Kbps}$ & Voice + data \\
\hline & PHS & 1995 & $1880-1930$ & $64 \mathrm{Kbps}$ & $20-40 \mathrm{Kbps}$ & Voice + data \\
\hline & CDMAOne & 1996 & $450 ; 800 ; 1700 ; 1900$ & $14.4 \mathrm{Kbps}$ & $7-10 \mathrm{Kbps}$ & Voice + data \\
\hline & iDEN & 2001 & $800 ; 900$ & $50-60 \mathrm{Kbps}$ & $20-30 \mathrm{Kbps}$ & Voice + data \\
\hline
\end{tabular}




\section{Access Comparison - CDMA}

Figure 4 shows CDMA multiple access technology is used in IS95 standard (Interim standards).

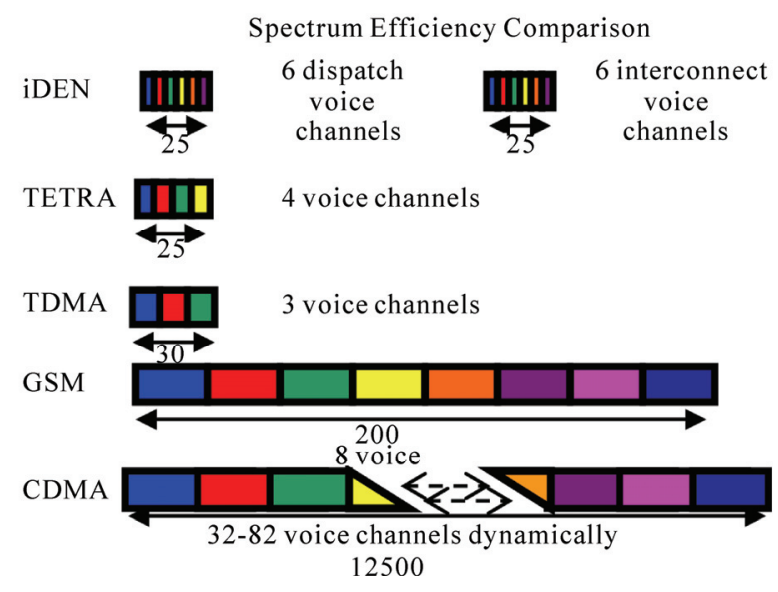

Figure 1. Spectrum efficiency comparison.

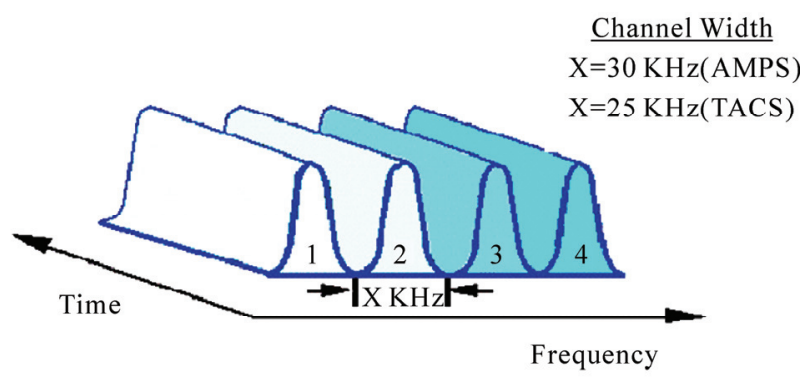

1 User per Narrowband Channel

Figure 2. FDMA.

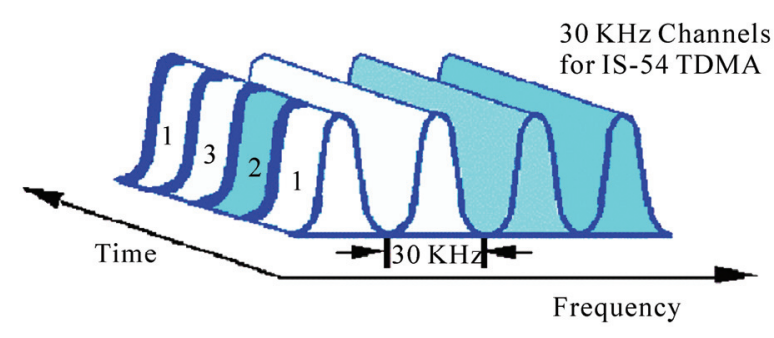

3 Users per Narrowband Channel

Figure 3. TDMA.

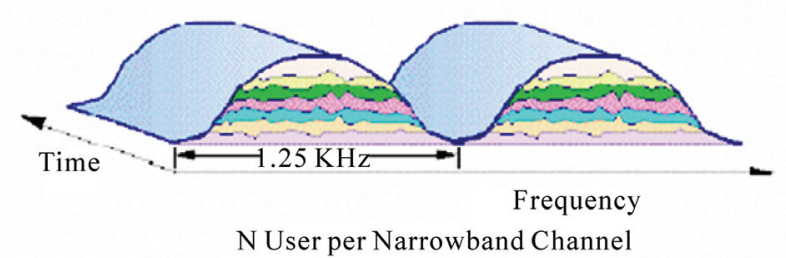

Figure 4. CDMA.
Each user is assigned a code during call setup which is multiplied or spread using a PN sequence, this results in a much wider signal than in other multiple access technologies. Wideband signals reduce interference and allow for 1 cell frequency re-use.

There is no time division which means all users use the entire carrier all of the time.

\subsection{GSM}

GSM (Global System for Mobile communication) belongs to the second mobile phone generation and was first established in 1992 in Europe. The users channels are separated on the one hand in the frequency domain using the frequency domain multiple access technique (FDMA) and on the other hand in the time domain using the time domain multiple access technique (TDMA). The time frame with the length $60 / 13 \mathrm{msec}$ is divided into 8 time slots which are assigned to different users. In addition the frequency band is divided into different channel each having $200 \mathrm{kHz}$ bandwidth. Adjacent base stations are not allowed to use the same frequencies.

Downlink and uplink signals have different frequency bands, so we are talking about the frequency domain duplex (FDD).

The Table 3 bellow show the characteristics of GSM.

The peak data rates achieved by GSM are $13 \mathrm{~kb} / \mathrm{s}$ for full rate speech encoded voice signal TCH/FS. Figure 5 shows how the modulation data are generated from the encoded speech data.

The speech data are generated by encoding the speech samples with duration of $20 \mathrm{msec}$ into 260 bit blocks. The data rate corresponds to $13 \mathrm{~kb} / \mathrm{s}$.

The 260 bits data blocks are divided into two classes:

Table 3. Table of GSM specification.

\begin{tabular}{cc}
\hline System Parameters & Specification \\
\hline Uplink frequency band & $890-915 \mathrm{MHz}$ \\
Downlink frequency band & $935-960 \mathrm{MHz}$ \\
Number of carriers/band & 125 \\
Multiple-access method & TDMA \\
Number of users/carrier & 8 \\
Data rate/carrier & $270.8 \mathrm{Kbps}$ \\
Speech-coding rate & $13 \mathrm{Khz}$ \\
Speech encoder & RPE-LPC \\
Coded-speech rate & $22.8 \mathrm{kbps}$ \\
Modulation & GMSK with BT $=0.30$ \\
Interleaver & Block \\
Frequency-hopping rate & 217 hops/sec \\
\hline
\end{tabular}




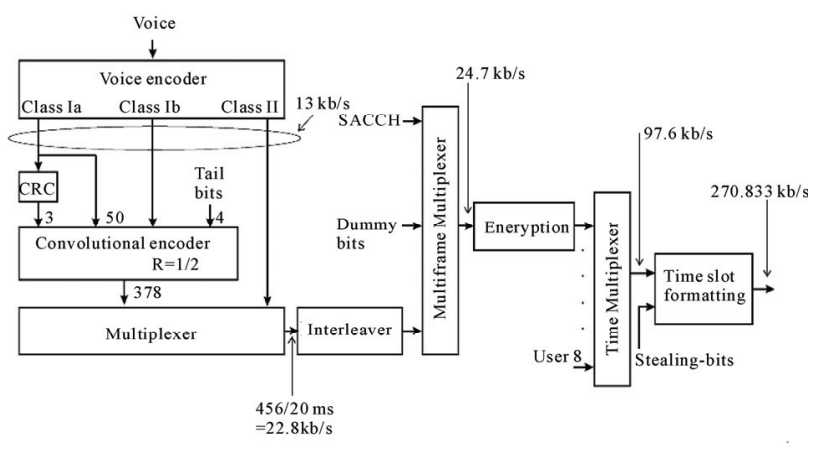

Figure 5. GSM modulation.

1) Class I: Includes 182 bits which are sensitive to bit errors and are considered as important. These bits are encoded using convolutional coding with the rate of $1 / 2$ and a constrain length 4 . The 182 bits are split into 50 bits (Class Ia) and 132 bits (Class Ib). Before convolutional encoding, 3 cyclic redundancy check bits (CRC) generated by the block encoder are added to the class Ia bits and 4 tail bits are added to the Class Ib bits.

2) Class II: Includes 78 bits which are less important and are transmitted without protection.

After channel coding the resulting 456 bits are interleaved and multiplexed with the slow associated control channel data (SACCH) and the dummy bits to build a multiframe with a time duration of $120 \mathrm{~ms}$. The multiframe includes 24 traffic channel frames, 1 idle frame and 1 slow associated control channel frame. After encryption, the different user frames are multiplexed to build a time frame with 8 time slots, each time slot includes in case of the traffic channel $2 \times 57$ bits. In the last step, the time slots are formatted to build the data bursts as show in Figure 6.

\subsection{CDMA IS-95a (CDMA One)}

Code Division Multiple Access (CDMA) was originally developed by Qualcomm; it is also referred to as CDMAOne and its standard designation IS-95A. This technology uses individual code designations to distinguish carrier channels in the spectrum from one another, in place of the frequency or time division systems in use by the technologies such as GSM. However, CDMA is similar to FDD systems such as GSM in that it uses one radio channel for sending information from base stations to end-user devices (downlink), and a separate channel for the uplink.

CDMA uses a $1.25 \mathrm{MHz}$ radio channel and support data rates of up to $14.4 \mathrm{kbps}$. Its architecture is based on a packetised backbone and its transport protocol is very similar to the computer transport network. Under this protocol, data information is split into packets. Each packet contains information data code and an overhead for error control, addressing and reassembly information that enable the network to organize information at the receiving end. CDMA efficiency depends on the number of users that concurrently communicate with the base station as these users compete for finite power provided by the transmitter. This phenomenon, known as cell breathing, significantly reduces the range of the communication network. In addition, CDMA does not benefit from the same roaming capabilities that have helped to make GSM the dominant mobile standard.

Figure 7 shows how the traffic signal is encoded and mapped into $20 \mathrm{~ms}$ frames before spreading, scrambling, and modulation.

After data coding and interleaving, the signal is encrypted with the long PN code. From each frame containing 384 encoded data bits, 16 are replaced by power control bits. The location of the power control bits is fixed by 4 bits derived from the PN code which is decimated by 6 to four times $800 \mathrm{kbps}$. The power control bit rate is also $800 \mathrm{kbps}$ and for each power control bit, 16 locations are possible. After Spreading and adding the other user and logical channel, the I and Q signals are generated by using two different real scrambling codes.

The data rate can be reduced after each frame from 9.6 kbps to $4.8 \mathrm{kbps}, 2.4 \mathrm{kbps}$ or $1.2 \mathrm{kbps}$ (rate set 1) by using symbol repetition after convolutional encoding. The mobile terminal must always support the rate set 1 , but may also support a second rate set (rate set 2), which is derived from the data rate $14.4 \mathrm{kbps}$ and include the rates

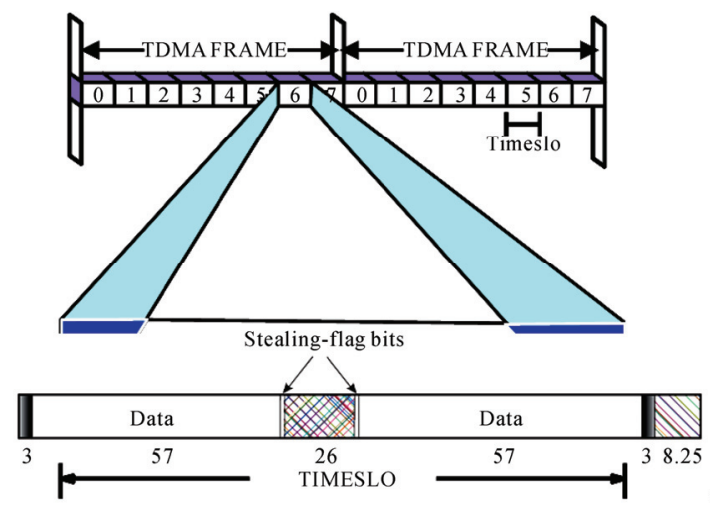

Figure 6. GSM burst.

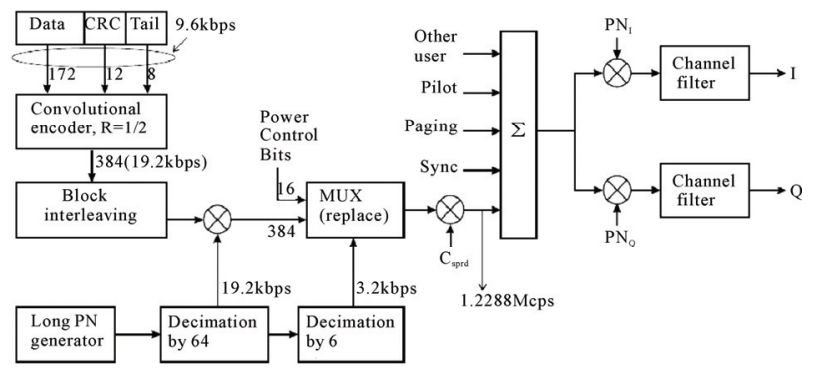

Figure 7. DMA modulation. 
$7.2 \mathrm{kbps}, 3.6 \mathrm{kbps}$ and $1.8 \mathrm{kbps}$. In the rate set 2, 2 of 6 bits have to be punctured before block interleaving.

\section{2.5 G Networks}

"Generation 2.5 " is a designation that broadly includes all advanced upgrades for the $2 \mathrm{G}$ networks.

Generally, a 2.5G GSM system includes at least one of the following technologies:

1) High-speed circuit-switched data (HSCSD)

2) General Packet Radio Services (GPRS)

3) Enhanced Data Rates for Global Evolution (EDGE).

An IS-136 system becomes $2.5 \mathrm{G}$ with the introduction of GPRS and EDGE, and an IS-95 system is called 2.5G when it implements IS-95B, or CDMA2000 1xRTT upgrades

\subsection{HSCSD}

HSCSD uses a maximum of four circuit-switched timeslots (each timeslot has throughput of $9.6 \mathrm{kbps}$ on 900 $\mathrm{MHz}$ networks, or $14.4 \mathrm{kbps}$ on $1800 \mathrm{MHz}$ networks) to increase data rates to a maximum of 38.4-57.6 kbps, provided that the timeslots are high speed data (HSD) capable. This technology requires a software upgrade for the GSM network infrastructure base.

\subsection{GPRS}

GPRS is a more radical step in the development of GSM towards higher data rate communication and was introduced into GSM networks as an intermediate step between $2 \mathrm{G}$ and $3 \mathrm{G}$. The implementation of GPRS allows a move away from circuit-switched data to the delivery of packet data based on GSM's TDMA technology. The packet switching nature of GPRS makes mobile data faster and cheaper and offers continuous connectivity and access to online services. Some operators have chosen to charge users as a function of data transmitted rather than connection time, as is the case for GSM voice calls, because GPRS uses network resources and bandwidth only when data is actually transmitted.

GPRS capability to provide a higher data rate relies on the different coding schemes defined in its specifications. There are four different coding schemes (Figure 8), each with a different level of error correction overhead. The level of overhead is inversely proportional to the available throughput; that is, the lower the channel protection, the higher and the throughput available. The maximum theoretical throughput - $171.2 \mathrm{kbps}$ - corresponds to the coding scheme 4 (CS-4)

\subsection{EDGE}

The main difference between GSM and EDGE consists on the data modulation which is based on 8-PSK instead of GMSK modulation used by GSM. In this way the data rate provided by EDGE is 3 times higher than GSM (up to $384 \mathrm{kbit} / \mathrm{s}$ ).

In each EDGE burst as shown in Figure 9, 346 coded data bits can be transmitted. Due to the lower symbol distance comparing to GSM, EDGE requires a better transmission quality. This can be achieved by reducing the cell size trough increasing the number of the base stations

\subsection{CDMA2000 $1 \times$ RTT (1X)}

The next phase of cdmaOne development was the Single Carrier Radio Transmission Technology $(1 \times$ RTT $)$ CDMA standard (increasingly known as just ' $1 \mathrm{X}$ '), an enhanced capability technology that provides increased capacity and data rates up to $144 \mathrm{kbps}$ for mobile devices.

The main difference to IS-95 is the use of complex scrambling and depending on the radio configuration; the data can be modulated using QPSK instead of BPSK before spreading. In case of downlink, 9 different radio configurations (RC1-RC9) are defined in cdma2000 standard. The five first radio configurations (RC1-RC5) are based on the spreading rate 1 and provide a maximum data rate of $307.2 \mathrm{kbps}$ (RC4) while the four last radio configurations (RC6-RC9) are based on the spreading rate 3 used in the multi-carrier technique. Figure 10 shows for the three different radio configurations (RC1, $\mathrm{RC} 2$ and $\mathrm{RC} 3$ ) the performed data processing steps between the user data and the IQ modulated signal.

\section{3G Networks}

The principal objectives of $3 \mathrm{G}$ networks are the delivery of higher data rate services with worldwide compatibility.

Coding parameters for the coding schemes

\begin{tabular}{|l|c|c|c|c|c|c|c|c|c|}
\hline Scheme & Code rate & USF & $\begin{array}{c}\text { Pre-coded } \\
\text { USF }\end{array}$ & $\begin{array}{c}\text { Radio } \\
\text { Block } \\
\text { excl.USF } \\
\text { and BCS }\end{array}$ & BCS & Tail & $\begin{array}{c}\text { Coded } \\
\text { bits }\end{array}$ & $\begin{array}{c}\text { Punctured } \\
\text { bits }\end{array}$ & $\begin{array}{c}\text { Data rate } \\
\text { kb/s }\end{array}$ \\
\hline CS-1 & $1 / 2$ & 3 & 3 & 181 & 40 & 4 & 456 & 0 & 9.05 \\
\hline CS-2 & $\approx 2 / 3$ & 3 & 6 & 268 & 16 & 4 & 588 & 132 & 13.4 \\
\hline CS-3 & $\approx 3 / 4$ & 3 & 6 & 312 & 16 & 4 & 676 & 220 & 15.6 \\
\hline CS-4 & 1 & 3 & 12 & 428 & 16 & - & 456 & - & 21.4 \\
\hline
\end{tabular}

Figure 8. GPRS coding schemes.

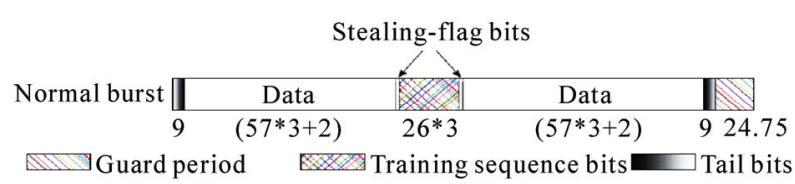

Figure 9. EDGE burst. 


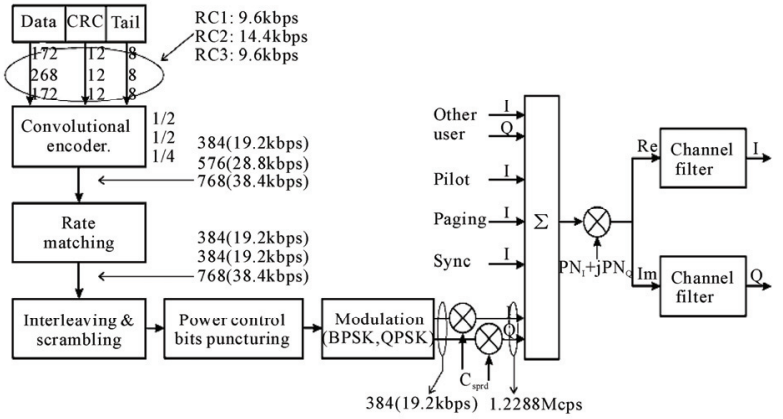

Figure 10. $1 \times$ RTT modulation.

The promise of new radio spectrum encouraged some of the world's mobile operators to pay very high prices for $3 \mathrm{G}$ licenses. Most $3 \mathrm{G}$ systems will operate in the less crowded IMT-2000 core band, operating at $2 \mathrm{GHz}$. This band has been largely dedicated to $3 \mathrm{G}$ services, promising licensees sufficient capacity for the network expansion necessary to host new value-added data services.

The main $3 \mathrm{G}$ networks currently deployed are based on WCDMA and EV-DO. A summary of some of the key features of the two systems is shown in Figure 11.

\section{Third Generation Partnership project (3GPP)}

The Third Generation Partnership project (3GPP) defines a range of standards including WCDMA, TDCDMA and refinements to GSM, GPRS and EDGE. 3GPP is a cooperation of ETSI (Europe), ATIS (North America), CCSA (China), TTA (South Korea) and ARIB/TTC (Japan) to create a global $3 \mathrm{G}$ standard that follows the ITU's IMT-2000 project.

3GPP technologies are based on the GSM evolution path and the identified $3 \mathrm{G}$ standard is now widely known as Universal Mobile Telecommunications System (UMTS). UMTS is the European/Japanese answer for $3 \mathrm{G}$ system standardisation and is sometimes referred to as $3 \mathrm{GSM}$, depicting the evolution of the GSM technologies towards a $3 \mathrm{G}$ standard.

3GPP standards include several hundred specifications and are categorized in Releases; Figure 12 lists the 3GPP standards defining the operation of $3 \mathrm{G}$ and beyond.

3GPP is generally seen as the most important standardisation body in the mobile segment and has defined many important systems, including WCDMA, HSDPA, HSUPA, TD-CDMA, TD-SCDMA and IMS. 3GPP is also in the process of defining Long Term Evolution (LTE), a standard that will attempt to specify systems that this report classifies as $3.9 \mathrm{G}$.

\section{GPP2}

3GPP2 was established in December 1998 and is collaboration between ARIB and TTC in Japan, CCSA in China, TIA in North America and TTA in South Korea. As with 3GPP, the aim of this standardisation group is to oversee the development of a global $3 \mathrm{G}$ standard. How

\begin{tabular}{llllll}
\hline System & Bandwidth & $\begin{array}{l}\text { Peak downlink } \\
\text { speeds }\end{array}$ & $\begin{array}{l}\text { Actual downlink } \\
\text { throughput }\end{array}$ & $\begin{array}{l}\text { Peak uplink } \\
\text { speeds }\end{array}$ & $\begin{array}{l}\text { Actual uplink } \\
\text { throughput }\end{array}$ \\
\hline WCDMA & $2 \times 5 \mathrm{MHz}$ & $2 \mathrm{Mbps}$ & $220-384 \mathrm{Kbps}$ & $384 \mathrm{Kbps}$ & $220-320 \mathrm{Kbps}$ \\
\hline EV-DO & $2 \times 1.25 \mathrm{MHz}$ & $2.4 \mathrm{Mbps}$ & $300-600 \mathrm{Kbps}$ & $2 \mathrm{Mbps}$ & $153 \mathrm{Kbps}$ \\
\hline
\end{tabular}

Figure 11.3G networks features.

\begin{tabular}{lrl}
\hline Release & Data frozen & Selected features \\
\hline 99 & Dec-99 & WCDMA,TD-CDMA \\
\hline 4 & Mar-01 & All-IP core network, TD-SCDMA \\
\hline 5 & Jun-02 & HSDPA,IMS phase 1 \\
\hline 6 & Mar-05 & HSUPA,IMS phase 2,MBMS \\
\hline 7 & Jun-07 & HSPA+with MIMO,EDGE Evolution,IMS VCC \\
\hline 8 & 2009 & LTE,SAE,All-IP Network \\
\hline
\end{tabular}

Figure 12. 3GPP releases.

ever, in practical terms, 3GPP2 is responsible for the standardisation of CDMA2000, a 3G technology based on CDMA standards which is primarily used in North America, South Korea and Japan.

\subsection{Wideband Code-Division Multiple Access (WCDMA)}

WCDMA is a 3 GPP and IMT-2000 standard derived from CDMA. It is a third-generation wireless network technology offering much higher data speeds to portable devices than most $2.5 \mathrm{G}$ networks.

WCDMA can support images, data and video communication at $384 \mathrm{Kbps}$ with full mobility, with a theoretical maximum of 2 Mbps for fixed and nomadic usage. WCDMA systems operate in a wide $5 \mathrm{MHz}$ channel, compared with the $200 \mathrm{KHz}$ carrier for GSM/GPRS, which is a basic reason why WCDMA delivers faster data rates and increased capacity when compared with previous networks.

WCDMA operators typically have allocations of between $20-40 \mathrm{MHz}(2 \times 10 \mathrm{MHz}$ up to $2 \times 20 \mathrm{MHz})$ of spectrum in the paired FDD bands of $1920-1980 \mathrm{MHz}$ (which is used for uplink) and $2110-2170 \mathrm{MHz}$ (downlink) as shown in Figure 13.

\subsection{CDMA2000 $1 \times$ EV-DO Release 0}

CDMA2000 $1 \times$ EV-DO Release 0 (EV-DO) was the first attempt to bring higher data rates to existing CDMA2000 $1 \times$ RTT networks. It allows data rates of up to $2.4 \mathrm{Mbps}$ in the downlink, but commercial implementations have

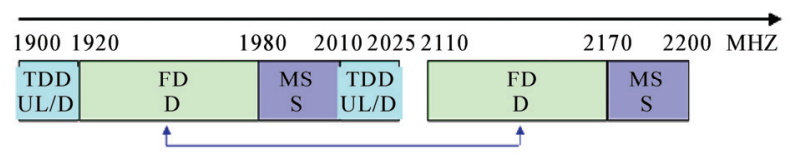

Figure 13. IMT frequency band. 
limited this to around 300-600 Kbps since several users have to be catered for Cdma2000 $1 \times$ EV-DO (Single Carrier Evolution-Data Optimized) is specified for the frequency bands which are only reserved for the data transmission. By using turbo coding in addition with 16-QAM and 8-PSK as modulation format, a maximum data rate of $2.4576 \mathrm{Mbps}$ and $1843.2 \mathrm{Mbps}$ can be achieved for downlink and uplink, respectively. The frame structure has been also changed in this substandards, the pilot and the control channels are no more transmitted in parallel using different spreading codes, they are time multiplexed with the traffic channel. From the time frame, with $1280 / 3 \mathrm{~ms}$ duration, $40 / 3 \mathrm{~ms}$ have been reserved for the control channel and the rest is used for the traffic channel.

The frame part reserved for the traffic channel is divided into 248 time slots which can be assigned to different users with variable data rates for time domain multiple accesses. Each time slot with the time duration of $1.667 \mathrm{~ms}$ include $4 \times 400$ data and preamble chips, $4 \mathrm{x}$ 64 MAC chips and $2 \times 96$ pilot chips. Figure 14 shows the forward channel structure including traffic channel coding, spreading, modulation, and time multiplexing of traffic, pilot, medium access control channel, and preamble bits.

\section{3.5G Networks}

The main objective of the $3.5 \mathrm{G}$ is to increase the throughput to about to $20 \mathrm{Mbits} / \mathrm{s}$, but in practice the data speed is about 1Mbps. The Figure 15 bellows summarize the most $3.5 \mathrm{G}$ with some characters.

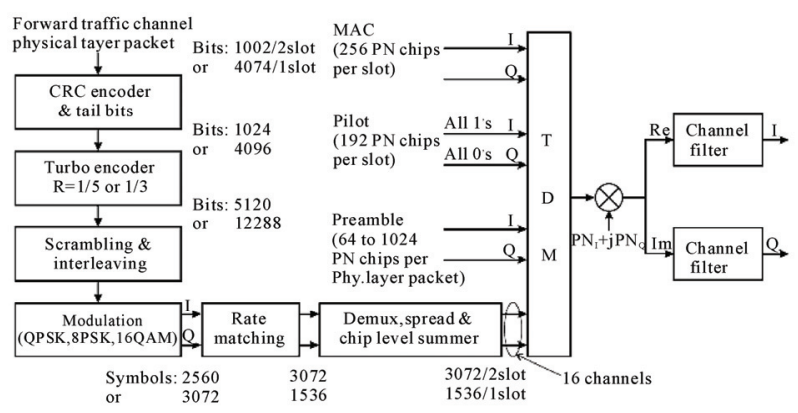

Figure 14. 1 × EV-DO coding.

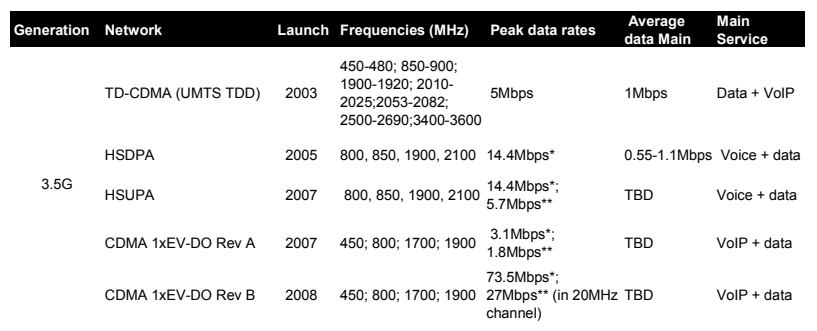

Figure 15. 3.5G networks.

\subsection{High Speed Downlink Packet Access (HSDPA)}

High Speed Downlink Packet Access (HSDPA) is a 3.5 $\mathrm{G}$ upgrade for existing WCDMA networks. Its key advantages are that it improves maximum downlink data rates to $14.4 \mathrm{Mbps}$, compared with $2 \mathrm{Mbps}$ for WCDMA, reduces latency to $100 \mathrm{~ms}$, compared with $180-200 \mathrm{~ms}$ for WCDMA, and can improve base station data capacity by a factor of five in dense urban environments HSDPA introduces a new transport channel - formally known as high-speed downlink shared channel (HS-DSC) - that uses a number of intelligent and adaptive techniques to improve performance.

Some of HSDPA's other key features include:

1) Adaptive Modulation and Coding (AMC)

By sending acknowledge reports from the CRC check to the base station, the base station can retransmit the data frame in case of incorrect transmission using a different puncture scheme (soft combining and incremental redundancy)

2) Hybrid Automatic Repeat Request (H-ARQ).

By using shorter radio frames with time duration of 2 ms equivalent to $3 \mathrm{~W}$-CDMA time slots and by moving the medium access control (MAC) to the node-B (BTS) rather than in the radio network controller (RNC), the coding and the modulation schemes can be adapted rapidly to the quality of the radio link using the fast scheduling function. In case of good transmission condition, 16-QAM can be used instead of QPSK. For the encoding, turbo-encoding with a fixed rate of $1 / 3$ is used and the coding adaptation is based on different rate-matching which affects the effective code rate. Figure 16 shows how the information data coding is performed.

\subsection{High Speed Uplink Packet Access (HSUPA)}

Though HSDPA includes higher data rates for downloading,

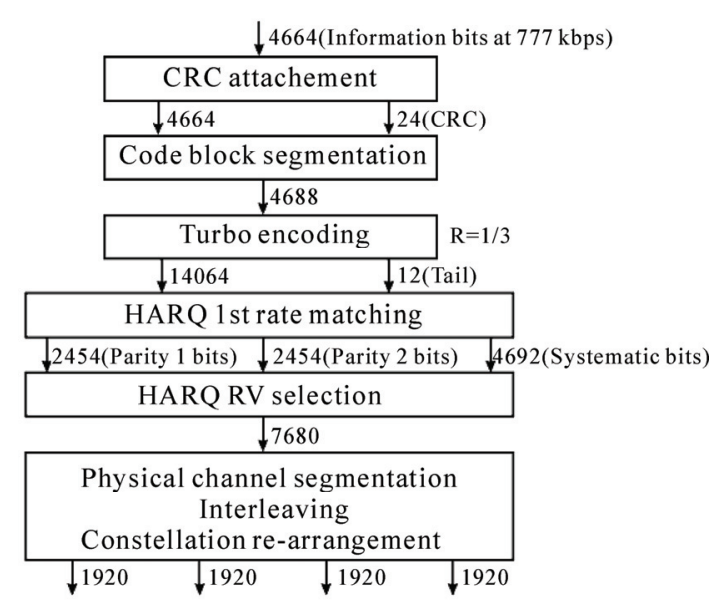

Figure 16. 3.5 HSDPA data coding. 
the upload data rates are still limited by the $3 \mathrm{G}$ system. As an evolutionary upgrade to HSDPA, High Speed Uplink Packet Access (HSUPA) brings a faster upload channel (up to $5.76 \mathrm{Mbps}$ ) in order to provide a more symmetric communication channel and allow full-duplex packet services to be used. HSUPA, which is also referred to as uplink enhanced dedicated channel (E-DCH), uses some of the same techniques as HSDPA but applies them to the uplink rather than downlink.

HSUPA was released as a part of 3GPP Release 6 during March 2005 and is now in the process of being demonstrated by infrastructure vendors around the world. Unlike HSDPA, HSUPA uses lower order modulation schemes in order to conserve battery life at the user terminal. Another reason for not including more advanced modulation schemes is that these may impose higher amplifier requirements at the terminal - which would mean bigger size and possibly more heat dissipation.

The Figure 17 shows that HSDPA reduces latency in the downlink, HSUPA reduces latency in the uplink, making the overall latency of the system (both downlink and uplink) acceptable for real-time applications including VoIP. VoIP services generally require a round-trip latency of $100 \mathrm{~ms}$ or less to provide acceptable quality, and networks with both HSUPA and HSDPA have RTT latency of around $50 \mathrm{~ms}$.

\subsection{CDMA2000 $1 \times$ EV-DO Revision A}

CDMA2000 1xEV-DO Revision A (EV-DOrA) is the 3 GPP2's version of $3.5 \mathrm{G}$. Building on the success of EV-DO Release 0, EV-DOrA brings higher data rates to both downlink and uplink and includes some significant improvements. EV-DOrA supports downlink data rates of up to $3 \mathrm{Mbps}$ and uplink rates of up to $1.8 \mathrm{Mbps}$, allowing for full duplex services to be used, including video communications and other multimedia-rich services.

Apart from the increase in data rates, the most important aspect of EV-DOrA is that an all-IP air interface is introduced. Consequently, VoIP services can be used over the mobile network accompanied by competitive

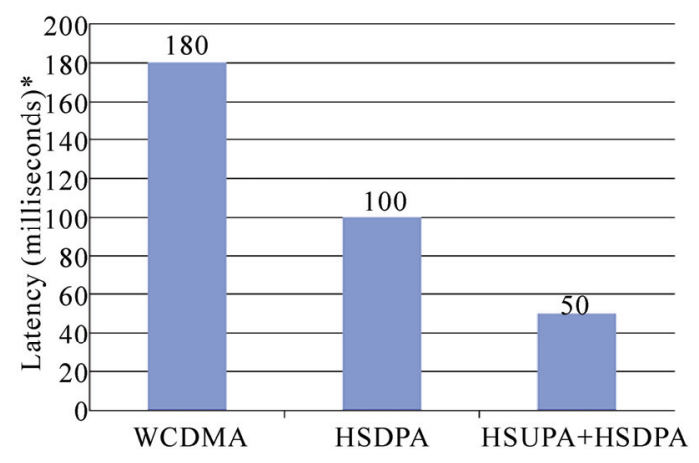

Figure 17. Delay comparison. data and multimedia services. Moreover, EV-DOrA allows QoS for low latency applications, multicast capabilities and higher system capacity..

\subsection{CDMA2000 1xEV-DO Revision B}

CDMA2000 $1 \times$ EV-DO Revision B (EV-DOrB) introduces even higher data rates for the communication link by aggregating frequency carriers to increase capacity. Overall, it is expected that there will be up to 15 carriers of $1.25 \mathrm{MHz}$ each within $20 \mathrm{MHz}$ of bandwidth.

EV-DOrB includes intelligent algorithms in the Medium Access Control (MAC) layer to optimise the communication link, much like HSDPA or any new communication network.

EV-DOrB also introduces new modulation schemes, the most significant of which is 64-QAM. This allows considerably higher data rates assuming that the channel enjoys very favorable conditions. In the case when a user is stationary or in the vicinity of the base station, higher data rates will be available, but as the user becomes mobile or is near the edge of the cell, 64-QAM will not be able to provide service and another - more robust modulation scheme will have to be used

\subsection{Time Division CDMA (TD-CDMA)}

Contrary to WCDMA, which uses frequency duplex (different frequencies are used for the uplink and downlink), TD-CDMA multiplexes both channels into different timeslots.

Therefore, it uses a single frequency channel and allows the operator to allocate bandwidth to the downlink or uplink. Although this may not be necessary in voice communications that require symmetric links, data communications usually require asymmetric links, with the downlink requiring much higher bandwidth than the uplink.

TD-CDMA is promoted by the UMTS TDD Alliance, which aims to provide a forum for development issues as well as to promote the UMTS TDD market environment. TD-CDMA provides several advantages compared with WCDMA. Higher data rates are available since resource allocation extends to the time domain and latency is also inherently improved. Initial implementations of TDCDMA systems have illustrated data rates of up to 5 Mbps and latency as low as $50 \mathrm{~ms}$, but practical applications are expected to be limited to $1 \mathrm{Mbps}$.

\section{3.9G Networks}

Both 3GPP and 3GPP2 refer to standards beyond 3.5G as Long-Term Evolution (LTE), although other terms including $3.9 \mathrm{G}$ are used. LTE is at a relatively early stage of development in both $3 \mathrm{GPP}$ and $3 \mathrm{GPP} 2$ so there is not 
a broad consensus within the industry on terminology, with some preferring to use other terms such as $3.99 \mathrm{G}$, Super $3 \mathrm{G}$ or Beyond $3 \mathrm{G}$. In this report Informa Telecoms $\&$ Media uses the terms $3.9 \mathrm{G}$ or LTE to refer to this group of emerging technologies and standards.

3.9G technologies aim to improve the performance of $3.5 \mathrm{G}$ networks significantly and to provide some backward-compatibility with those networks. In particular, $3.9 \mathrm{G}$ networks are being designed to use $3 \mathrm{G}$ spectrum allocations, as opposed to $4 \mathrm{G}$ systems, which will require new spectrum.

\section{WIMAX Evolution}

WiMAX, which stands for Worldwide Interoperability for Microwave Access, is a global effort to evolving fixed-wireless technology to support mobility, which will bring WiMAX into competition with $3 \mathrm{G} / 3.5 \mathrm{G}$ technologies such as WCDMA/HSDPA and EV-DO/EVDOrA.

The 802.16 standard family is defined by the IEEE. The vision has changed a lot in the last 10 years:

1) 1998: IEEE formed 802.16 group to develop a standard for a wireless metropolitan area network (MAN) as fixed wireless access

2) 2004: Standards ratified 802.16 and two bands has been defined $10-66 \mathrm{GHz}$ for LOS, and $2-11 \mathrm{Ghz}$ for NLOS.

3) 2005: The standard 802.16 e is completed to allow mobility application in the band $2-6 \mathrm{GHz}$

4) 2006: The product is certified with WIMAX as commercial name.

WIMAX is started as fixed broadband now it is really the first $3.9 \mathrm{G}$ network.

\section{4G Networks}

Fourth-generation (4G) networks are still at an early stage of development - so early in fact that there is no industry consensus on the definition of $4 \mathrm{G}$. However, the ITU has developed a recommendation for an initial working definition of 'Advanced Technology Evolution', its term for $4 \mathrm{G}$ (Figure 18) which can be summarised as follows:

1) 4G systems should be IP-based and should combine

\begin{tabular}{lllll}
\hline Fcaturcs & 3G & 3.5G & 3.9G & 4G \\
\hline Spectrum & 3G FDD & 3G FDD \& TDD & 3G FDD \& TDD & New spectrum \\
\hline Radio aspects & WCDMA & HSDPA,HSUPA,TD-CDMA & OFDMA,MIMO & OFDMA,MIMO and others \\
\hline Bandwidth & $5 \mathrm{MHz}$ & $5 \mathrm{MHz}$ & $1.25-20 \mathrm{MHz}$ & $100 \mathrm{MHz}$ \\
\hline Data rate & $0.384-2 \mathrm{Mbps}$ & $144.4 \mathrm{Mbps}$ & $30-100 \mathrm{Mbps}$ & $100-1000 \mathrm{Mbps}$ \\
\hline Network switching & Circuit and packet & Circuit and packet & Packet only & Packet only \\
\hline
\end{tabular}

Figure 18. 4G requirements.

elements of mobile, wireless and fixed networks in a seamless architecture transparent to the user.

2) Target data rates should be $100 \mathrm{Mbps}$ for mobile users and 1 Gbps for nomadic users.

3) Worldwide common spectrum and open global standards should be pursued.

\section{Conclusions}

The architecture of $4 \mathrm{G}$ systems is also likely to be design to deliver the long-held industry vision of seamless access to services across multiple mobile, wireless and fixed networks. This could be enabled by a range of technologies including IMS, IPv6, OFDMA, MIMO.etc. The 4G networks should also be planned and optimized to support these different services (mobiles and fixes) with differents QoS.

\section{References}

[1] M. Roberts and S. Sherrington, Future Mobile Broadband, 2007.

[2] CDMA Developer Group, 23 July 2007. http://www.cdg.org

[3] "Cingular Wireless: Spectrum Efficiency Comparison, GSM vs. UMTS vs. 1 × RTT," Research Material, 14 March 2002.

[4] "Mobile Applications \& Operating Systems," 3rd Edition, 17 October 2006.

[5] "World Cellular Information Service," August 2007.

[6] "Radio Transmission and Reception," Technical Specification Group GSM/EDGE Radio Access Network, 3rd Generation Partnership Project, 2005.

[7] "User Equipment Radio Transmission and Reception (FDD)," Technical Specification Group Radio Access Network, 3rd Generation Partnership Project, 2005. 